\title{
75. CONTRIBUCIÓN AL CONOCIMIENTO DE LAS XENÓFITAS EN ESPAÑA: CATÁLOGO PROVISIONAL DE LA FLORA ALÓCTONA DE ALMERÍA
}

\author{
Elías DANA, Manuel I. CERRILLO, Mario SANZ ELORZA, \\ Eduardo SOBRINO y Juan F. MOTA
}

Contribution to the knowledge about xenophytes in Spain: Provisional check-list of alien flora in Almeria.

Palabras clave. Biodiversidad, invasiones biológicas, especies alóctonas, sureste ibérico.

Key words. Biodiversity, biological invasions, exotic species, South-eastern Iberian Peninsula.

La introducción e intercambio de especies vegetales entre regiones geográficas diferentes es un proceso que, a pesar de tener sus orígenes en los albores de la humanidad, ha adquirido una mayor trascendencia en los últimos tres siglos, coincidiendo con el auge de los viajes transoceánicos.

La Península Ibérica no ha sido una excepción a este proceso generalizado de alteración del elenco florístico de los territorios, y, al menos parcialmente, es sabido que en España existe un buen número de táxones xenófitos o alóctonos (Sanz-Elorza et al., en prensa). En numerosos países se posee un razonable conocimiento de la contribución de la flora alóctona en el catálogo global de especies. En muchos casos (Estados Unidos, Australia, etc.) existen bases de datos informatizadas en las que se recoge no sólo una lista de especies, sino también información cartográfica o de tipo ecológico (fenología, comportamiento invasor, etc.). Sin embargo, en España y hasta la fecha, es muy escasa la información disponible al respecto. Además, con frecuencia, es difícil de extraer, ya que las especies alóctonas suelen ser citadas en el seno de otros trabajos más amplios en los que se listan las especies presentes en territorios concretos. Por último, aún se carece de un listado global de especies alóctonas a partir del cual se pueda extraer información que pueda resultar de utilidad, tanto desde el punto de vista puramente científico como para diseñar proyectos de prevención y manejo de estas especies y de los ecosistemas afectados.

Con objeto de contribuir a rellenar este vacío, se ha creado recientemente el grupo de trabajo sobre Especies Alóctonas, Urbanas y Cuarentenas de la Sociedad Española de Malherbología, que tiene, entre otros objetivos fundamentales, la creación de una base de datos nacional en la que se recoja la información disponible relativa a las xenófitas (Sobrino et al., 1999). El objetivo de este artículo se enmarca en este proyecto, y reside en la elaboración de un catálogo preliminar donde se encuentren recogidas las especies alóctonas presentes en la provincia de Almería (fig. 1).

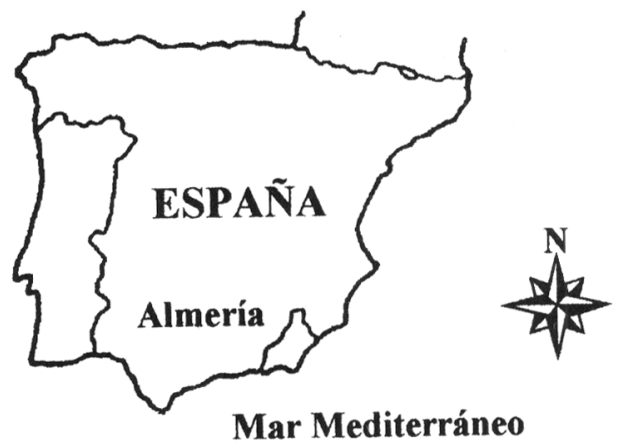

Figura 1. Localización del área de estudio. Study site. 
Además del catálogo que aportamos, el presente trabajo supone una contribución en cuanto a la sistematización del tratamiento para el grado en que las especies alóctonas estudiadas se encuentran integradas en los ecosistemas de la región. El listado provisional de xenófitas se ha elaborado a partir de la información bibliográfica proporcionada por Castroviejo et al. (1986-1999), Dana et al. (1998; 1999a; 1999b), Fernández et al. (1991), OrtegaOlivencia et al. (1986) y Sagredo (1987). Los datos relativos a su carácter invasor proceden tanto de las referencias que en estas obras se hacen a la provincia de Almería, como de la experiencia de campo de los autores, mientras que los datos que las acompañan -origen, biotipo, modo de introducción-se obtuvieron considerando también otras obras generales (Valdés et al., 1987; Dimitri, 1988).

Para la asignación de los xenotipos se ha seguido a Kornás (1978) y a Pysek et al. (1995). A falta de una cartografía más precisa sobre el grado de expansión de las especies alóctonas en Almería, la distinción de xenotipos recoge sólo si la especie en cuestión es diáfito, metáfito o si, de acuerdo con la bibliografía manejada, no se le conoce un comportamiento invasor en la provincia. De acuerdo con Sanz-Elorza (2001) y Sanz-Elorza et al. (en prensa), en el primer caso, la especie, aunque tiene potencialidad para escaparse y formar poblaciones auto-perdurables, aún no se ha naturalizado de manera efectiva, mientras que en el segundo, el taxon en cuestión está plenamente integrado y aparece de forma constante en una región, propagándose de manera autónoma, comportándose como si de una especie autóctona se tratara. Las categorías corológicas son una combinación adaptada de las propuestas por Mateo \& Crespo (1990) y por Takhatajan (1986), mientras que los biotipos siguen a Raunkiaer (1934).

El total de especies (200 entre invasoras y no invasoras) registradas en este estudio puede parecer elevado a primera vista, ya que constituyen cerca del $6-8 \%$ de la flora total estimada para la provincia (Sagredo, 1987). El número de táxones citados en conjunto para la provincia es sensiblemente mayor (Dana et al., 1999a) pero en algunos casos aún no se posee información confirmada sobre diversas características de las comentadas en este trabajo, mientras que en otros, su presencia o estado taxonómico necesita ser aclarado.

Con los datos disponibles hasta el momento, el número de especies alóctonas con algún comportamiento invasor (121 especies, de las que 73 pueden ser consideradas como metáfitos y 48 como diáfitos) supone en torno al $4-5 \%$ de la flora estimada para Almería, por lo que resulta sensiblemente inferior al encontrado en otras localidades receptoras de flora xenófita. Así, aunque ciertas zonas de Almería pueden actuar como acumuladoras de xenófitas (suponen el $22 \%$ de las especies registradas en la capital, Dana et al., 1999b), cuando se comparan estos porcentajes con los encontrados por diversos autores en otras zonas geográficas podemos concluir que la importancia del componente alóctono en la región estudiada no es muy elevada, al menos en cuanto al número de especies. Por ejemplo, Sanz-Elorza \& Sobrino (1999) indican que las especies alóctonas en una zona de la comarca del Baix Camp (Tarragona) contribuyen con $20 \%$ al total de especies de la zona, mientras que Natali \& Jeanmonod (1996) han constatado que el $17 \%$ de la flora de Córcega está compuesta por especies alóctonas. En un contexto geográfico más amplio, como el continente europeo, el porcentaje total de especies introducidas es mucho mayor, alcanzando el 13\% (Weber, 1997) (fig. 2). El porcentaje encontrado para la provincia de Almería está más próximo al valor medio encontrado en diversas zonas continentales mediterráneas, como el $5.8 \%$ encontrado para la flora de Friuliu-Venezia Giulia en Italia (Martini \& Poldini, 1995) o el 5.4\% de la provincia de Huesca (Sanz-Elorza, 2001). 


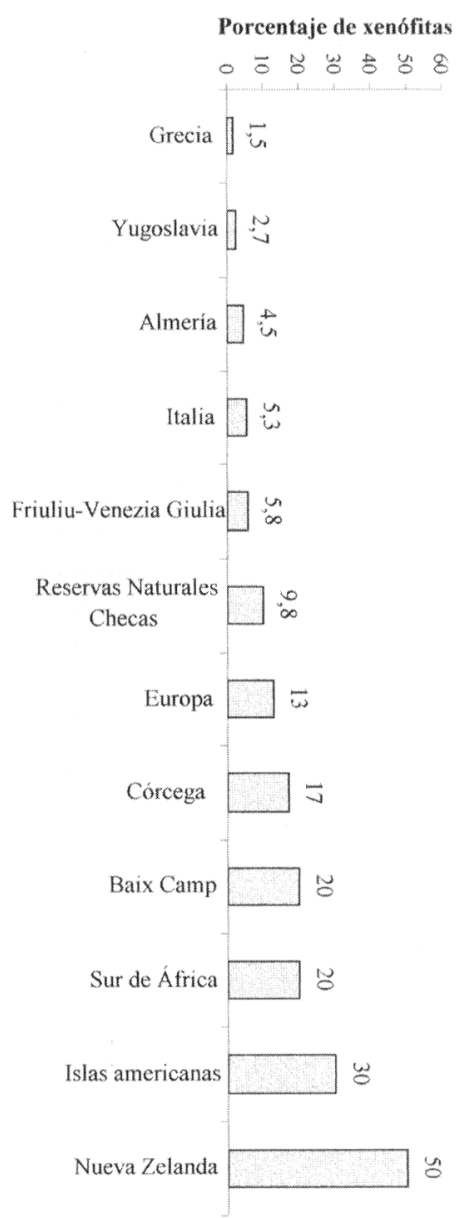

Figura 2. Porcentaje de especies alóctonas (incluyendo arqueófitos y neófitos) de distintas regiones geográficas del Globo. Percentage of alien taxa (including archaeophytes and neophytes) in different geographical regions of the World.

En el contexto mundial (fig. 2), este porcentaje resulta aún más reducido; así, en diversas islas norteamericanas de clima mediterráneo se han encontrado porcentajes muy elevados que oscilan en torno al $30 \%$ (Rejmanek et al., 1991), mientras que en la flora del sur de África, con unas 20 mil especies nativas, se ha detectado ya la presencia de un millar (20\% aprox.) de táxones alóctonos (Wells, 1991). En las regiones tropicales del
Globo, colonizadas en los últimos dos siglos, los porcentajes son mucho más elevados. Así, Heywood (1989) cita que el porcentaje de plantas alóctonas en la flora de Nueva Zelanda asciende al $50 \%$, mientras que Mauchamp (1997) cifra la importancia de las alóctonas más o menos naturalizadas en un $42.2 \%$ suponiendo ésta una de las amenazas más graves a los ecosistemas nativos. Para la realización de la figura 2 se han empleado las siguientes fuentes: Heywood (1989), Nueva Zelanda; Wells (1991), Sur de África; Rejmanek et al. (1991), valores medios obtenidos de numerosas islas norteamericanas; Martini \& Poldini (1995), Friuliu-Venezia Giulia -Italia-; Kucera (1995), media para las reservas naturales checas; Natali \& Jeanmonod (1996), Córcega; Weber (1997), media europea; Sanz-Elorza \& Sobrino (1999) -Baix Camp, Tarragona-. Para el cálculo de los porcentajes de alóctonas en Grecia y en Italia, se han empleado las estimaciones para el número de especies vegetales aportados por Strid \& Tan (1991) y por Davis et al. (1994) respectivamente y los datos aportados por Weber (1997). Para todos los datos en los que se manejan rangos y no cifras netas, se ha calculado el valor medio para la elaboración de la gráfica comparativa.

Las razones por las que la provincia de Almería presenta porcentajes globales de alóctonas tan reducidos aún no han sido exploradas y aclaradas definitivamente pero su representatividad depende del tipo de hábitat considerado (Dana et al., en prensa). Los ambientes extremos -como las zonas áridas y semiáridas y las altimontanas- han sido considerados generalmente como poco invasibles, o al menos, con un reducido contingente alóctono (Baker, 1986; Casasayas, 1990). Sin embargo, no se ha demostrado aún definitivamente si la menor invasibilidad en este tipo de territorios se debe a que éstos y otros ambientes extremos no han sido tan intensamente alterados como otros sistemas más propicios para el asentamiento humano, o 
a que sus biocenosis cuentan con una especial resistencia a la invasión por especies alóctonas (Rejmanek et al., 1991). En el caso de la provincia de Almería, estudios paralelos indican que a gran escala, es bastante probable que la severidad ambiental de buena parte del sureste ibérico y las peculiaridades sociales de la región hayan actuado como filtros que han limitado las posibilidades de éxito de las especies foráneas introducidas (Dana et al, datos no publicados).

En cualquier caso, es interesante resaltar que de las especies que se estudian en este trabajo, sólo 49 sólo pueden considerarse como diáfitos. Los motivos por los que estas especies no han llegado a naturalizarse de manera efectiva pueden ser muy diversos (menor número de eventos de introducción por parte del ser humano, inadaptación de las especies al medio, escasez o ausencia de polinizadores o hábitats apropiados, fenómenos competitivos con otras especies, etc.) y requieren un estudio pormenorizado para cada especie en concreto, ya que los procesos de invasión de los ecosistemas son, en gran medida, probabilísticos, y dependen, no sólo de los rasgos autecológicos específicos, sino también de las condiciones puntuales en las que se encuentra el medio en el momento de la recepción de cada especie en cuestión (Williamson, 1996; Rejmanek, 1996a). En cualquier caso, estos diáfitos son los táxones sobre los que resultaría más factible aplicar técnicas de control sobre sus poblaciones o bien regular su futuro uso con distintos fines ornamentales, alimenticios, etc.-. Las restantes especies, o bien se encuentran ampliamente distribuidas, o bien no han presentado hasta ahora comportamiento invasor. Del último contingente, habría que prestar una especial atención a aquellos táxones de los que se tienen noticias acerca de su comportamiento invasor en otros países como por ejemplo Lantana camara, Melia azederach, Prunus spp., Pinus radiata, Pinus strobus, Ligustrum spp., etc. (e.g. Henderson, 1995; 1998a; 1998b; Lavergne et al., 1999), ya que no se puede descartar que inicien procesos de colonización a medio-largo plazo en la región.

Las especies para las que se ha obtenido información se listan a continuación.

\section{Aceraceae}

Acer negundo L. N América. Macrofanerófito. Forestal, Ornamental. Diáfito.

\section{Agavaceae}

Agave americana L. Neotropical. Hemicriptófito rosulado. Textil. Metáfito.

Agave furcroydes Lem. Neotropical. Hemicriptófito rosulado. Textil; plantación para obtener fibra (600 Ha.) en el S de Almería. Metáfito.

Agave sisalana Engelm. Neotropical. Hemicriptófito rosulado. Textil; plantación para obtener fibra (600 Ha.) en el S de Almería. Metáfito.

\section{Aizoaceae}

Aptenia cordifolia (L. fil.) Schwantes. Capense. Caméfito sufruticoso. Ornamental. Diáfito.

Carpobrutus acinaciformis (L.) L. Bolos. Capense. Caméfito sufruticoso. Ornamental, fijadora de taludes, dunas y terrenos. Diáfito.

Carpobrutus chilensis (Molina) N. E. Br. Neotropical. Caméfito sufruticoso. Ornamental. Diáfito.

Carpobrutus edulis (L.) N. E. Br. Capense. Caméfito sufruticoso. Ornamental y fijadora de terrenos. Diáfito a Metáfito.

\section{Amaranthaceae}

Amaranthus albus L. N América. Terófito escaposo. Accidental. Metáfito.

Amaranthus blitoides S. Watson. N América. Terófito escaposo. Accidental. Metáfito.

Amaranthus blitum L. ssp. blitum. Paleosubtropical. Terófito escaposo. Accidental. Metáfito.

Amaranthus cruentus L. N América. Terófito escaposo. Ornamental en la Península Ibérica, y también como hortaliza o seudocereal en otras zonas del Mundo. Diáfito.

Amaranthus deflexus L. Neotropical. Terófito / 
Hemicriptófito escaposo. Accidental. Metáfito. Amaranthus gracilis Desf. Neotropical. Terófito escaposo. Accidental. Metáfito.

Amaranthus graecizans L. ssp. gracizans. Paleosubtropical. Terófito escaposo. Accidental. Metáfito.

Amaranthus graecizans L. ssp. silvestris (Vill.) Bre. Incierto, ya que mientras que es Paleosubtropical para Mateo \& Crespo (1990), para Castroviejo et al. (1990), probablemente es nativa del Mediterráneo. Terófito escaposo. Accidental. Metáfito.

Amaranthus hybridus L. Neotropical. Terófito escaposo. Accidental. Metáfito.

Amaranthus muricatus (Moq.) Gillies ex Hicken. Neotropical. Hemicriptófito escaposo. Accidental. Metáfito.

Amaranthus retroflexus L. N América. Terófito escaposo. Accidental. Metáfito.

Amaranthus viridis L. Neotropical. Terófito / Hemicriptófito escaposo. Accidental. Metáfito.

\section{Anacardiaceae}

Pistacia vera L. Paleotropical. Mesofanerófito. Alimentario y Ornamental. Sin comportamiento invasor.

Schinus molle L. Neotropical. Macrofanerófito. Ornamental. Diáfito.

Schinus terebinthifolia Raddi. Neotropical. Mesofanerófito. Ornamental. Diáfito.

\section{Apiaceae}

Coriandrum sativum L. W Asia. Terófito escaposo. Alimentario. Diáfito.

Petroselinum crispum (Miller) A. W. Hill. Iranoturaniana. Hemicriptófito bienal. Alimentario. Sin comportamiento invasor conocido en la provincia, aunque sí en otros lugares. No obstante, su estatus necesita aclaración.

\section{Apocinaceae}

Lochnera rosea (L.) Reichenb. Tropical. Caméfito fruticoso. Ornamental. Sin comportamiento invasor.

\section{Araceae}

Colocasia esculenta (L.) Schott. Paleotropical.
Geófito rizomatoso. Ornamental; en algunas regiones del Globo para alimentación (por los rizomas tuberosos). Sin comportamiento invasor.

Monstera deliciosa Liebm. Neotropical. Fanerófito escaposo. Ornamental. Sin comportamiento invasor.

Zantedeschia aethiopica (L.) Sprengel. Capense. Geófito rizomatoso. Ornamental. Sin comportamiento invasor.

\section{Asclepiadaceae}

Araujia sericifera Brot. Neotropical. Fanerófito escaposo. Ornamental. Diáfito. Quizás incluso Metáfito, ya que su carácter invasor ha sido repetidamente demostrado en otras regiones, por lo que es necesario aclarar su estatus.

\section{Basellaceae}

Bousingaultia cordifolia Ten. Neotropical. Fanerófito escaposo. Ornamental. Metáfito.

\section{Boraginaceae}

Heliotropium curassavicum L. Neotropical. Caméfito reptante. Accidental. Metáfito.

\section{Buddlejaceae}

Buddleja davidii Franchet. Chinojaponesa (China). Nano/Meso fanerófito cespitoso. Ornamental. Hasta ahora no hay referencias que indiquen que esta especie presenta un comportamiento invasor en la provincia, donde ha comenzado a emplearse recientemente en parques y jardines. No ocurre así en otras zonas de España (Sanz-Elorza et al., en prensa) o del Occidente Mediterráneo (e.g. Natali \& Jeanmonod, 1996), donde esta especie parece asilvestrarse con relativa facilidad. Por ello es necesario realizar un seguimiento del posible comportamiento invasor de esta especie en la región.

\section{Cactaceae}

Opuntia maxima Miller. Neotropical. Fanerófito suculento. Alimentario (frutos) y formación de setos; en otros tiempos cultivada para la cría de cochinillas. Metáfito. 
Opuntia subulata (Mühlenpfordt) Engelm. Neotropical. Fanerófito suculento. Ornamental. Diáfito (localmente con apariencia de Metáfito).

\section{Cannaceae}

Canna indica L. Neotropical. Geófito rizomatoso. Ornamental. Sin comportamiento invasor.

\section{Caryophyllaceae}

Gypsophila elegans Bieb. Iranoturaniana. Terófito escaposo. Ornamental. Diáfito.

\section{Casuarinaceae}

Casuarina cunninghamiana Miq. Australia. Fanerófito. Ornamental. Sin comportamiento invasor.

Casuarina stricta Aiton. Australia. Fanerófito. Ornamental. Sin comportamiento invasor.

\section{Chenopodiaceae}

Atriplex semibaccata R. Br. Australia. Hemicriptófito escaposo. Forrajera. Metáfito.

Chenopodium ambrosioides L. Neotropical. Hemicriptófito escaposo. Medicinal (cultivada intensamente como vermífuga). Metáfito.

Chenopodium multifidum L. Neotropical. Hemicriptófito escaposo. Accidental. Metáfito.

\section{Compositae}

Aster squamatus (Sprengel) Hieron. Neotropical. Hemicriptófito bienal. Accidental. Metáfito.

Bidens aurea (Aiton) Sherff. Neotropical. Hemicriptófito escaposo. Accidental. Metáfito.

Bidens bipinnata L. Neotropical. Terófito escaposo. Accidental. Metáfito.

Bidens pilosa L. Neotropical. Terófito escaposo. Accidental. Metáfito.

Conyza albida Willd. ex Spreng. Neotropical. Terófito escaposo. Accidental. Metáfito.

Conyza bonariensis (L.) Cronq. Neotropical. Terófito escaposo. Accidental. Metáfito.

Conyza canadensis (L.) Cronq. N América. Terófito escaposo. Accidental. Metáfito.

Senecio mikanoides Otto ex Walpers. África. Fanerófito escandente. Ornamental. Sin comportamiento invasor.
Xanthium spinosum L. Neotropical. Terófito escaposo. Accidental. Metáfito.

\section{Convolvulaceae}

Ipomoea batatas (L.) Lam. Neotropical. Geófito tuberoso. Alimentario. Sin comportamiento invasor.

Ipomoea purpurea (L.) Rothm. Neotropical. Terófito escandente. Ornamental. Sin comportamiento invasor en la región, aunque sí en otras provincias.

Ipomoea sagittata Poiret. Subtropical. Geófito rizomatoso. Ornamental. Diáfito.

\section{Crassulaceae}

Aeonium arboreum (L.) Webb \& Berth. Marruecos. Caméfito INanofanerófito suculento. Ornamental. Diáfito.

\section{Cruciferae}

Coronopus didymus (L.) Sm. Neotropical. Terófito rosulado. Accidental. Metáfito.

\section{Cucurbitaceae}

Citrullus colocynthis (L.) Schrad. Iranoturaniana. Hemicriptófito reptante. Medicinal. Metáfito.

Cucumis melo L. Paleotropical. Terófito reptante. Alimentario. Generalmente sin comportamiento invasor, pero puede aparecer como Diáfito (ergasiófito).

Cucumis sativus L. Paleosubtropical. Terófito reptante. Alimentario. Generalmente sin comportamiento invasor, pero puede aparecer como Diáfito (ergasiófito).

Cucurbita pepo L. Incierto. Terófito reptante. Alimentario. Sin comportamiento invasor.

Lagenaria siceraria (Molina) Stand. Paleotropical. Terófito escandente. Alimentario. Sin comportamiento invasor.

\section{Cupressaceae}

Cupressus arizonica E. L. Greene. N América. Macrofanerófito. Ornamental y forestal. Diáfito. 
Cupressus sempervirens L. E Mediterráneo. Macrofanerófito. Ornamental y forestal. Diáfito. Platycladus orientalis (L.) Franco. Chinojaponesa (China). Mesofanerófito. Ornamental y forestal. Sin comportamiento invasor claro.

\section{Cyperaceae}

Cyperus rotundus. Subtropical. Geófito rizomatoso. Accidental. Metáfito.

\section{Ebenaceae}

Diospyros kaki L. Fil. Chinojaponesa. Macrofanerófito. Alimentario. Sin comportamiento invasor.

\section{Euphorbiaceae}

Chamaesyce nutans Lag. Neotropical. Terófito escaposo. Terófito reptante. Accidental. Metáfito.

Chamaesyce serpens (Kunth) Small. Neotropical. Terófito reptante. Accidental. Metáfito.

Euphorbia lathyris L. Iranoturaniana (China y C Asia). Hemicriptófito bienal. Accidental. Metáfito.

Ricinus communis L. Paleotropical. Mesofanerófito. Alimentario (actualmente ornamental, antiguamente alimentario). Metáfito.

\section{Geraniaceae}

Pelargonium grandiflorum Willd. Capense. Caméfito sufruticoso. Ornamental. Sin comportamiento invasor.

Pelargonium peltatum (L.) Aiton. Capense. Caméfito sufruticoso. Ornamental. Diáfito (ergasiófito).

Pelagornium zonale (L.) Aiton. Capense. Caméfito sufruticoso. Ornamental. Diáfito (ergasiófito).

\section{Gramineae}

Arundo donax L. Asia. Geófito rizomatoso. Motivo de introducción incierto. Metáfito.

Cortaderia selloana (Schultes) Ascherson \& Graebner. Neotropical. Hemicriptófito escaposo. Ornamental. En la región, aparentemente, sin comportamiento invasor.
Echinochloa colonum (L.) Link. Paleotropical. Terófito escaposo. Accidental; mala hierba de cultivo. Metáfito.

Paspalum paspalodes (Michx.). Subtropical. Geófito rizomatoso. Accidental; mala hierba de cultivo. Metáfito.

Paspalum urvillei Steudel. Neotropical. Geófito rizomatoso. Accidental y forrajera (introducida como forrajera en Portugal). Metáfito.

Paspalum vaginatum Swartz. Subtropical. Geófito rizomatoso. Accidental. Metáfito.

Polypogon monspeliensis (L.) Desf. Paleotropical. Terófito escaposo. Accidental. Metáfito.

Saccharum officinarum L. Paleotropical. Hemicriptófito cespitoso. Alimentario. Sin comportamiento invasor.

Secale cereale L. Iranoturaniana. Terófito escaposo. Hemicriptófito cespitoso. Alimentario. Diáfito.

Setaria geniculata (Lam.) Beauv. N América. Hemicriptófito rizomatoso. Accidental. Metáfito.

Setaria pumila (Poiret) Schultes \& Schultes fil. Paleotropical. Terófito escaposo. Accidental. Metáfito.

Setaria viridis (L.) Beauv. Paleotropical. Terófito escaposo. Accidental. Metáfito.

Stenotaphrum secundatum (Walter) O. Kuntze. Tropical/subtropical. Geófito rizomatoso. Ornamental (céspedes; usada como forrajera en Argentina). Metáfito.

Zea mays L. Neotropical. Terófito escaposo. Alimentario. Diáfito.

\section{Hydrangeaceae}

Hydrangea macrophylla (Thumb.) Ser. Chinojaponesa. Nanofanerófito/Mesofanerófito. Ornamental. Sin comportamiento invasor.

Philadelphus coronarius L. Incierto. Probablemente SE Europa y Caúcaso (Castroviejo et al. 1997). Mesofanerófito. Ornamental. Diáfito.

\section{Iridaceae}

Iris albicans Lange. Procede de Arabia según Valdés et al. (1987). Geófito rizomatoso. Ornamental, se cultiva ampliamente en la Región Mediterránea, especialmente en los cementerios musulmanes. Diáfito.

Iris germanica L. Incierto (Sagredo, 1987; Mateo \& Crespo, 1990), mientras que según Valdés et al. 
(1987) probablemente procede del Este del Mediterráneo. Geófito rizomatoso. Ornamental, posible origen híbrido. Diáfito.

\section{Juglandaceae}

Juglans nigra L. N América. Macrofanerófito. Ornamental y forestal. Sin comportamiento invasor.

Juglans regia L. Iranoturaniana. Macrofanerófito. Alimentario, ornamental, forestal (madera) y farmacéutico. Sin comportamiento invasor.

\section{Labiatae}

Mentha spicata L. Incierto. Hemicriptófito escaposo. Alimentario y Ornamental. Sin comportamiento invasor aparente.

Mentha $x$ piperita L. Incierto. Hemicriptófito escaposo. Alimentario y Ornamental. Sin comportamiento invasor aparente.

Ocimum basilicum L. Paleotropical. Terófito escaposo. Cultivada (alimentario y medicinal). Sin comportamiento invasor aparente.

\section{Leguminosae}

Acacia cyclops A. Cunn. ex. G. Don. f. Australia. Mesofanerófito. Ornamental, repoblaciones. Metáfito.

Acacia dealbata Link. Australia. Macrofanerófito. Ornamental. Sin comportamiento invasor en la provincia, aunque sí en otras regiones españolas.

Acacia farnesiana (L.) Willd. Neotropical (Antillas). Mesofanerófito. Ornamental. Metáfito.

Acacia retinoides Schlechtendal. Australia. Mesofanerófito. Ornamental. Metáfito.

Acacia saligna (Labill.) Wendland. Australia. Mesofanerófito. Ornamental. Metáfito.

Albizzia lophanta Benth. Australia. Nanofanerófito /Mesofanerófito. Ornamental. Diáfito.

Cercis siliquastrum L. Europa meridional y Asia Occidental. Mesofanerófito escaposo. Ornamental. Sin comportamiento invasor conocido en la provincia, pero sí se ha constatado si comportamiento como diáfito en otras zonas mediterráneas (e.g. Natali \& Jeanmonod, 1996).

Cicer arietinum L. Probablemente S Asia. Terófito escaposo. Cultivada como alimento. Sin comportamiento invasor.
Gleditsia triacanthos L. N América. Mesofanerófito. Ornamental. No se conoce que se comporte como invasora en la provincia, aunque sí en otras zonas de España. Su estatus necesita de posterior confirmación.

Lens culinaris Medicus. Iranoturaniana (W Asia). Terófito escandente. Alimenticio. Diáfito.

Medicago sativa L. Incierto. Hemicriptófito escaposo. Forrajero. Metáfito.

Mimosa pudica L. Neotropical. Terófito / Hemicriptófito escaposo. Ornamental. Sin comportamiento invasor.

Phaseolus lunatus L. N América. Terófito escandente. Alimentario. Sin comportamiento invasor.

Pisum sativum ssp. sativum L. C Asia. Terófito escaposo. Alimentario. Diáfito.

Robinia hispida L. N América. Caméfito fruticoso. Ornamental. Sin comportamiento invasor. Citada por Sagredo (1987) como introducida en fase experimental, pero habría que confirmar su existencia actual en la provincia.

Robinia pseudoacacia L. N América. Macrofanerófito. Ornamental y forestal. Metáfito.

Sophora japonica L. Chinojaponesa (China). Macrofanerófito. Ornamental. Sin comportamiento invasor.

Wisteria floribunda (Willd.) DC. Chinojaponesa. Fanerófito escandente. Ornamental. Sin comportamiento invasor.

Wisteria sinensis (L.) Poir. N América. Fanerófito escandente. Ornamental. Sin comportamiento invasor.

\section{Liliaceae}

Aloe arborescens Miller. Capense. Nanofanerófito. Ornamental. Diáfito.

Aloe brevifolia Miller. Capense. Caméfito sufruticoso. Ornamental. Sin comportamiento invasor.

Aloe perfoliata L. Capense. Caméfito sufruticoso. Ornamental. Sin comportamiento invasor conocido.

Aloe variegata $\mathrm{L}$. Capense. Caméfito sufruticoso. Ornamental. Diáfito.

Aloe vera (L. fil.) Burm. Africa tropical (África y Arabia). Caméfito sufruticoso. Ornamental. Diáfito. 
Asparagus plumosus Baker. Capense. Fanerófito escaposo. Ornamental. Diáfito.

Lilium candidum L. Paleotropical (Asia). Geófito bulboso. Ornamental. Diáfito.

Lilium longiflorum Thunb. Chinojaponesa (Japón). Geófito bulboso. Ornamental. Sin comportamiento invasor.

Phormium tenax J. R. \& Fors. Nueva Zelanda. Hemicriptófito rosulado. Ornamental. Introducido en otras zonas del Globo para la fabricación de textiles y licores. Sin comportamiento invasor.

Yucca aloifolia L. N y C América. Mesofanerófito. Ornamental. Sin comportamiento invasor claro.

\section{Linaceae}

Linum usitatissimum L. Desconocido para Mateo \& Crespo (1990) y posiblemente oriunda de Asia según Dimitri (1988). Terófito escaposo. Textil y alimentario (aceite de lino). Metáfito.

\section{Malvaceae}

Alcea rosea L. Incierto. Hemicriptófito escaposo. Ornamental. Metáfito.

Hibiscus rosa-sinensis L. Chinojaponesa (China). Nanofanerófito /Mesofanerófito. Ornamental. Sin comportamiento invasor.

Lagunaria patersonii G. Don. Australia. Mesofanerófito /Macrofanerófito. Ornamental. Sin comportamiento invasor.

\section{Meliaceae}

Melia azederach L. Iranoturaniana. Macrofanerófito. Ornamental. Diáfito.

\section{Moraceae}

Broussonetia papyrifera (L.) Vent. Chinojaponesa. Mesofanerófito. Ornamental. Estado de naturalización dudoso, ya que Castroviejo et al. (1993) no la consideran naturalizada excepto en algunos puntos del litoral NO de España, mientras que Sagredo (1987) la considera en vías de expansión. La consideramos aquí como Diáfito a falta de más información.

Ficus elastica Roxb. Paleotropical. Macrofanerófito. Ornamental. Sin comportamiento invasor.
Morus alba L. Chinojaponesa. Macrofanerófito. Ornamental y alimentario (infrutescencias moras-) y textil (hojas para Bombyx mori L.). Se plantó profusamente en algunas regiones como la Sierra Nevada Almeriense o la huerta murciana. Diáfito.

\section{Musaceae}

Musa cavendishii Lamb. ex Paxton. Tropical/ Subtropical. Hemicriptófito escaposo. Ornamental y alimentario. Sin comportamiento invasor.

\section{Myoporaceae}

Myoporum tenuifolium G. Fors. Terófito Australia. Mesofanerófito. Ornamental. Diáfito.

\section{Myrtaceae}

Eucalyptus camaldulensis Dehnh. Australia. Macrofanerófito. Forestal. Metáfito.

Eucalyptus globulus ssp. globulus Labill. Australia. Macrofanerófito. Forestal. Diáfito (o incluso Metáfito según Castroviejo et al. 1997). Su estatus necesita de aclaración.

Eucalyptus gomphocephala DC. Australia. Macrofanerófito. Forestal. Diáfito (o incluso Metáfito según Castroviejo et al. 1997). Su estatus necesita de aclaración.

\section{Nyctaginaceae}

Mirabilis jalapa L. Neotropical. Geófito bulboso. Ornamental. Metáfito.

\section{Oleaceae}

Jasminum nudiflorum Lindley. Chinojaponesa. Nanofanerófito /Mesofanerófito. Ornamental. Sin comportamiento invasor.

Jasminum officinale L. Incierto. Probablemente SO Asia. Nanofanerófito. Ornamental. Sin comportamiento invasor.

Ligustrum lucidum Ait. China. Mesofanerófito / Macrofanerófito. Ornamental. Sin comportamiento invasor.

Ligustrum ovalifolium Hasskarl. Japón. Nanofanerófito /Mesofanerófito. Ornamental. Sin comportamiento invasor. 
Syringa vulgaris L. SE Europa (Rumanía y Península Balcánica). Mesofanerófito. Ornamental. Diáfito.

\section{Onagraceae}

Fuchsia speciosa Hort. Neotropical. Caméfito. Ornamental. Sin comportamiento invasor.

\section{Oxalidaceae}

Oxalis corymbosa DC. Neotropical. Geófito bulboso. Accidental. Metáfito.

Oxalis pes-caprae L. Capense. Geófito bulboso. Accidental. Metáfito.

\section{Palmae}

Chamaerodea elegans Mart. Neotropical. Fanerófito. Ornamental. Sin comportamiento invasor.

Oreodoxa regia H. B. K. Neotropical. Macrofanerófito. Ornamental. Sin comportamiento invasor.

Phoenix canariensis Chabaud. Macaronesia. Macrofanerófito. Ornamental. Sin comportamiento invasor.

Phoenix dactilifera L. Paleotropical. Macrofanerófito. Diversos usos: ornamental y alimentario, sombra, etc. Metáfito.

Trachycarpus fortunei (Hook.) H. Wendi. Chinojaponesa (China). Macrofanerófito. Ornamental. Sin comportamiento invasor.

Washingtonia filifera (Linden) H. Wendl. N América. Macrofanerófito. Ornamental. Sin comportamiento invasor.

Washingtonia robusta $\mathrm{H}$. Wendl. N América (México). Macrofanerófito. Ornamental. Sin comportamiento invasor.

\section{Papaveraceae}

Papaver somniferum L. Incierto. Terófito escaposo. Medicinal y alimentario (semillas con aceite usado en alimentación, repostería y usos industriales). Metáfito.

\section{Phytolaccaceae}

Phytolacca dioica L. Neotropical. Macrofanerófito. Ornamental. Diáfito.

\section{Pinaceae}

Larix decidua Miller. C Europa. Macrofanerófito. Ornamental, forestal. Sin comportamiento invasor.

Picea abies subsp. abies (L.) Karsten. N y C Europa. Macrofanerófito. Ornamental y forestal (repoblaciones en el $\mathrm{N}$ España). Sin comportamiento invasor.

Pinus canariensis Swet ex Sprengel. Macaronesia. Macrofanerófito. Forestal y ornamental. Sin comportamiento invasor.

Pinus pinea L. Probablemente Mediterráneo oriental. Macrofanerófito. Forestal y alimentario. Metáfito.

Pinus radiata D. Don. N América. Macrofanerófito. Ornamental, repoblación. Sin comportamiento invasor.

Pinus strobus L. N América. Macrofanerófito. Forestal. Sin comportamiento invasor.

Pinus sylvestris L. Eurosiberiana. Macrofanerófito. Forestal. Metáfito.

Pinus uncinata Ramond ex DC. Medit. W (Alpes, Pirineos y C España). Macrofanerófito. Forestal. Metáfito.

\section{Platanaceae}

Platanus hispanica Miller ex Münchh. Híbrido ( $P$. occidentalis $\times$ P. orientalis) (Castroviejo et al. 1990). Macrofanerófito. Ornamental, como especie forestal para repoblaciones en ríos y como árbol de sombra. Diáfito.

\section{Polygonaceae}

Polygonum orientale L. Paleotropical. Terófito escaposo. Ornamental. Diáfito.

\section{Portulacaceae}

Portulaca oleracea L. ssp. papillatostellulata Danin \& H. G. Baker. N y C América. Terófito reptante. Accidental (cultivos de verano).Metáfito.

\section{Proteaceae}

Grevillea robusta Cunninghan. Suráfrica. Macrofanerófito. Ornamental y Forestal. Sin comportamiento invasor. 


\section{Punicaceae}

Punica granatum L. Iranoturaniana. Mesofanerófito. Alimentario; usada también para formación de setos y ornamental. Diáfito-metáfito. Su estatus necesita aclaración.

\section{Rosaceae}

Cydonia oblonga Miller. Incierto (probablemente W Asia). Nanofanerófito/Mesofanerófito. Cultivada por sus frutos. Diáfito.

Eriobotrya japonica (Thumb.) Lindl Chinojaponesa. Macrofanerófito. Alimentario. Sin comportamiento invasor.

Malus domestica (Borkh.) Borkh. Iranoturaniana. Macrofanerófito. Alimentario. Diáfito.

Prunus cerasus L. Iranoturaniana. Mesofanerófito. Alimentario. Sin comportamiento invasor.

Prunus domestica L. Euroasiática. Macrofanerófito. Alimentario. Sin comportamiento invasor.

Prunus dulcis (Mill.) D. A. Webb. Iranoturaniana. Mesofanerófito. Alimentario. Usado también como ornamental. Aunque localmente pueda parecer Metáfito, lo consideramos por el momento Diáfito hasta poseer más información. En algunas zonas donde se ha cultivado aparece asilvestrado. Es necesario confirmar el mantenimiento de sus poblaciones en el tiempo.

Prunus persica (L.) Batsch. Chinojaponesa (China). Macrofanerófito. Alimentario. Sin comportamiento invasor.

Prunus triloba Lindl. Chinojaponesa (China). Mesofanerófito. Ornamental. Sin comportamiento invasor.

\section{Salicaceae}

Populus deltoides Marshall. N América. Macrofanerófito. Ornamental y forestal (repoblaciones en ríos). Metáfito.

Populus euphratica Olivier. Paleosubtropical. Macrofanerófito. Ornamental y forestal (en ríos). Citado por Sagredo (1987) -quien no comenta su estado- pero no por Castroviejo et al. (1993).

Populus nigra L. Paleotemplado (Mateo \& Crespo, 1990), o bien E de Europa Occidental y W de Asia (Castroviejo et al., 1993). Macrofanerófito. Forestal (protección riberas), ornamental. Metáfito.
Populus $x$ canadensis Moench. Artificial. Macrofanerófito. Forestal (ríos). Metáfito.

Salix babylonica L. Chinojaponesa (C y N China). Macrofanerófito. Ornamental (probablemente); quizás también cultivado para cestería. Diáfito.

Salix fragilis L. Incierto. Crece de forma natural en Europa y SW de Asia; es dudoso su carácter autóctono en España. Macrofanerófito. Cestería (mimbres), y a veces como ramón (forraje). Metáfito.

\section{Sapindaceae}

Cardiospermum haliacabum L. Tropical. Terófito escandente. Ornamental. Sin comportamiento invasor.

Koelreuteria paniculata Lam. Chinojaponesa (China). Macrofanerófito. Ornamental. Sin comportamiento invasor.

\section{Simaroubaceae}

Ailanthus altissima (Miller) Swingle. Chinojaponesa. Mesofanerófito / Macrofanerófito. Motivo de introducción desconocido por el momento. En algunas ciudades mediterráneas ha sido introducido como ornamental y de sombra. Metáfito.

\section{Solanaceae}

Cestrum parqui L'Hér. Neotropical. Nanofanerófito. Ornamental. Sin comportamiento invasor.

Datura inoxia Miller. Neotropical. Terófito escaposo. Accidental. Metáfito.

Datura stramonium L. Neotropical. Terófito escaposo. Accidental. Metáfito.

Nicotiana glauca R. C. Graham. Neotropical. Mesofanerófito. Accidental. Metáfito.

Solanum bonariense L. Neotropical. Nanofanerófito. Ornamental. Metáfito.

Solanum melongena L. Paleotropical. Terófito escaposo. (Caméfito fruticoso en su forma silvestre). Alimentario. Sin comportamiento invasor.

Solanum pseudocapsicum L. Neotropical. Caméfito sufruticoso. Ornamental. Sin comportamiento invasor.

Solanum tuberosum L. Neotropical. Geófito tuberoso. Alimentario. Sin comportamiento invasor. 


\section{Sterculiaceae}

Brachychiton diversifolium $\mathrm{R}$. Br. Australia. Macrofanerófito. Ornamental. Sin comportamiento invasor.

\section{Tropaeolaceae}

Tropaeolum majus L. Neotropical. Terófito reptante. Ornamental. Diáfito.

\section{Verbenaceae}

Lantana camara L. Neotropical. Nanofanerófito / Mesofanerófito. Ornamental. Diáfito (ergasiófito) incipiente.

Lippia triphylla (L'Hér.) O. Kuntze. Neotropical. Nanofanerófito /Mesofanerófito. Ornamental y medicinal. Sin comportamiento invasor.

\section{Vitaceae}

Parthenocissus quinquefolia (L.) Planchon. N América. Fanerófito escandente. Ornamental. Sin comportamiento invasor.

\section{Zygophyllaceae}

Zygophyllum fabago L. Iranoturaniana. Caméfito sufruticoso. Accidental. Metáfito.

\section{BIBLIOGRAFÍA}

BAKER, H.G. - 1986- Patterns of plant invasions in North America. In: H.A. Mooney, \& J.A. Drake (eds.) Ecology of Biological invasions of North America and Hawaii, pp. 44-57. SpringerVerlag, New York.

CASASAYAS, T. -1990- Widespread adventive plants in Catalonia. In: J.A.Hansen \& M. Debussche (eds.) Biological Invasions in Europe and the Mediterranean Basin, pp. 85-104. Kluwer Academic Publishers, Dordrecht. The Netherlands.

CASTROVIEJO, S. et al. -1986/1999- (eds.) Flora Ibérica. Plantas Vasculares de la Península Ibérica e Islas Baleares. Real Jardín Botánico de Madrid. CSIC. Madrid.

DANA, E., J. CABELLO, J.F. MOTA y J. PEÑAS -
1998- Acerca de tres especies nitrófilas en la provincia de Almería. Acta Bot. Malacitana 23: 252-256.

DANA, E., J.F. MOTA, S. VIVAS, M. SANZ \& E. SOBRINO -1999a- The alien flora of southeastern spain. An advance for the exotic plants database national research project. Proceedings $5^{\text {th }}$ International Conference on the Ecology of Invasive Alien Plants. La Maddalena, Sardinia (Italy), 13-16 october, 1999, pág. 49.

DANA, E., J. CABELLO, J.F. MOTA, M. CUETO y J. PEÑAS -1999b- Flora urbanícola de la ciudad de Almería: estudio florístico, fitocenológico y autoecológico. Monog. Fl. y Veg. Béticas 11: 133-149.

DANA, E., S. VIVAS \& J.F. MOTA -En prensa-. Urban vegetation of Almería City -a contribution to urban ecology in Spain. Landscape and Urban Planning.

DAVIS, S.D., V.H. HEYWOOD \& A.C. HAMILTON (eds.) -1994-Centres of plants diversity, Vol I. Europa, África, SW Asia and middle East. WWF-UICN-EC-ODA.

DIMITRI, M.J. -1988- Enciclopedia Argentina de Agricultura y Jardinería. Tomo I, vol. II. Ed. ACME S.A.C.I. Buenos Aires.

FERNÁNDEZ, C., M. CRUZ, M. LÓPEZ-PULIDO, C. AMEZCUA y D. CASADO -1991- Flora de Andalucía Oriental. Catálogo bibliográfico de las plantas vasculares. Universidad de Jaén. Jaén.

HENDERSON, L. -1995- Plant Invaders of Southern African. Agricultural Research Council. Pretoria

HENDERSON, L. -1998- Southern African plant invaders atlas (SAPIA) Applied Plant Science 12(1): $31-32$

HENDERSON, L. -1998- Invasive alien woody plants of the southern and southwestern Cape region, South Africa. Bothalia 28 (1): 91-112

HEYWOOD, V.H. -1989- Patterns, extents and modes of invasions of terrestrial plants. In: J.A. Drake \& H.A. Mooney (eds.) Biological invasions, a global perspective, pp. 31-55. John Wiley \& Sons, New York, NY.

KORNÁS, J. -?- Remarks on the analysis of a synanthropic flora. Acta Bot. Slov. Acad. Sci. Slov. Ser. A., 3: 385-393.

KUCERA, T. -1995-Zmeny flóry v maloplosnych chránenych územich. Zpr. Ces. Bot. Spolec. 30, Mater. 12: 137-140. 
LAVERGNE, C., J-C. RAMEAU, \& J. FIGIER -1999The invasive woody weed Ligustrum robustum subsp. walkeri threatens native forests on $\mathrm{La}$ Réunion. Biological Invasions 1: 377-392

MARTINI, F. \& L. POLDINI -1995- The hemerophytic Flora of Friuli-Venezia Giulia (N.E. Italy). Flora Mediterranea 5: 229-246

MATEO, G. y B. CRESPO -1990- Claves para la flora de Valencia. Ed. del Cenia al Segura. Valencia.

MAUCHAMP, A. -1997- Threats from alien plant species in the Galapagos Islands. Conservation Biology 11(1): 260-263.

NATALI, A. \& D. JEANMONOD -1996- Flore analytique des plantes introduites en Corse. Complèments au Prodrome de la Flore Corse. Conservatoire et Jardin botaniques. Génève.

ORTEGA-OLIVENCIA, A. y A.B. ROBLES - 1986Estudio botánico de las plantas adventicias en los cultivos extratempranos del litoral almeriense. In: F. Pascual, A. Ortega-Olivencia \& A.B. Robles, Plantas e insectos perjudiciales en invernaderos, pp. 7-145. Diputación de Almería. Almería.

PYSEK, P. 1995. On the terminology used in plant invasion studies. In P. Pysek, K. Prach, M. Rejmánek \& M. Wade (eds.), Plant InvasionsGeneral Aspects and Special Problems, pp. 7181. SPB Academic Publishing. Amsterdam. The Netherlands.

RAUNKIAER, C. -1934- The life forms of plants and statistical plant geography. Oxford. Clarendon Press.

REJMANEK, M., C.D. THOMSEN \& I.D. PETERS -1991- Invasive vascular plants of California In: R.H. Groves \& F. di Castri (eds.) Biogeography of Mediterranean Invasions, pp. 81-101. Cambridge University Press. Cambridge.

REJMÁNEK, M. -1996a- Species richness and resistance to invasions. Ecological Studies 122: 153-172

SAGREDO, R. -1987- Flora de Almería. Plantas vasculares de la provincia. Instituto de Estudios Almerienses. Diputación Provincial. Almería.

SANZ-ELORZA, M. \& E. SOBRINO -1999Diferencias en la capacidad de acogida de elementos florísticos alóctonos entre las zonas costeras y del interior en el mediterráneo occidental. Actas del VII Congreso de la
Sociedad Española de Malherbología, pp. 8388. Logroño.

SANZ-ELORZA, M. -2001- Flora y vegetación arvense y ruderal de la provincia de Huesca. Tesis Doctoral, inédita. Universidad de Lérida. SANZ-ELORZA, M., E. SOBRINO \& E. DANA 2001-. Análisis de xenótipos de la flora alóctona de la provincia de Huesca. Actas del Congreso 2001 de la Sociedad Española de Malherbología. [en prensa]

SANZ-ELORZA, M., SOBRINO, E. \& E. DANA En prensa-. Listado de plantas alóctonas invasoras en España (Península y Baleares). Lazaroa.

SOBRINO, E., M. SANZ-ELORZA, C.ZARAGOZA y E. DANA - 1999- La flora alóctona española: banco de datos. Actas del VII Congreso de la Sociedad Española de Malherbología, pp. 39 46. Logroño.

STRID, A. \& K. TAN -1991- Mountain flora of Greece. Vol 2. Edinburgh University press.

TAKHATAJAN, A. -1986- Floristic regions of the world. University of California Press. California.

VALDÉS, B., S. TALAVERA y E. FERNÁNDEZGALIANO - 1987- Flora Vascular de Andalucía Occidental, vols. 1-3. Ketres ed. Barcelona.

WEBER, E.F. - 1997- The alien flora of Europe: a taxonomic and biogeographic review. Journal of Vegetation Science 8: 565-572.

WELLS, M.J. - 1991- Introduced plants of the fynbos biome of South Africa. R.H. Groves \& F. di Castri (eds.) Biogeography of Mediterranean Invasions, pp. 115-129. Cambridge University Press. Cambridge.

WILLIAMSON, M. 1996. Biological Invasions. Chapman \& Hall, London.

Aceptado para su publicación en junio de 2001

Dirección de los autores. E. Dana, M.I. Cerrillo y J.F. Mota: Departamento de Biología Vegetal y Ecología Facultad de Ciencias Experimentales, Universidad de Almería. La Cañada de San Urbano s/n. E-04120. Almería. España. E-mail: edana@ual.es; M. Sanz Elorza y E. Sobrino: Departamento de Producción Vegetal. Botánica. Escuela Técnica Superior de Ingenieros Agrónomos. Universidad Politécnica de Madrid. Ciudad Universitaria s/n, 28040. Madrid 